\title{
Automated Gravity Terrain Corrections Using Satellite Stereo-pairs
}

\author{
Dr. Robert Davies ${ }^{1}$, Dr. Mark O'Neill ${ }^{2}$ and Dr. Rob McDonald ${ }^{1}$ \\ TerraDat (UK) Ltd' : PO Box 319, Cardiff, CF1 3UJ, UK \\ University of Oxford, $\mathrm{UK}^{2}$
}

By using SPOT stereo-pairs it is possible to automate the extraction of conjugate pairs of (matched) image co-ordinates. Propagation of these data through an appropriate sensor model, constrained by at least three ground control points, produces irregularly distributed height estimates which provide the basis of two elevation models. The first is a DEM of orthogonal grid points which is generated by Kriging. The second is a Delaunay triangular integrated network (TIN), which provides a more economic description of the terrain. This TIN can be used to calculate terrain corrections to any station co-ordinates that lie within the overlapping part of the imagery by repeated summation of the analytic triangular prism approximation. However, an unconstrained Delaunay triangulation leads to undesirable triangles that straddle ridges and valleys. Form-lines, that follow these features and avoid this effect, can be extracted by suitable filters from the gridded elevation data. Furthermore, just as triangular facets are a better approximation to terrain than rectangular prisms with horizontal surfaces, it is possible to approximate the elevation within quadrilaterals using bicubic spline functions. In this case, an analytic solution to the volume integration could not be found and so a Monte-Carlo approximation to point masses was used for the case of prisms that lie on the terrain surface. Initial tests revealed significant limitations of the MonteCarlo approximation method which has lead to the development of an improved algorithm which creates more regularly distributed point masses.

Apart from the selection of suitable ground control points, the entire DEM generation to terrain correction process can be automated, leading to correction errors of less than $10 \mu \mathrm{gals}$ in mountainous regions.

\section{INTRODUCTION}

The need for rapid and reliable determination of terrain corrections for gravity data is common to many regional scale exploration programs where significant topographic variation is encountered. Generally, most of this work is carried out using digitised contour maps and software based on the simple Hammer chart method (Hammer, 1982). This approach is both time consuming and prone to significant error, particularly in areas where high resolution maps are not available or the terrain is irregular. The approach we describe is semi-automated and requires only manual selection of suitable ground control points (minimum of three) that are identifiable on both satellite images and maps.

Digital elevation model (DEM) data are created using an advanced stereo-matching algorithm (O'Neill and Denos, 1996) and satellite or air-photography data. The chosen ground control points 
are used to cross reference the stereo-pair images and to fix the calculated DEM to a chosen coordinate system and known elevation level.

Density and DEM data are input to the terrain correction software (T-CORR) together with the known or planned location of the gravity survey stations. The output terrain correction for each gravity station is derived from the input density information and the entire elevation dataset at the resolution of the DEM from the near station to regional area.

\section{METHODOLOGY}

\section{$\underline{\text { DEM Generation }}$}

The most powerful method of generating digital elevation models (DEMs) is by stereo-matching satellite data. At present, however, there are few algorithms that can stereo-match greater than $90 \%$ of the input data. The process requires two stereo-images such as generated by the SPOT 1-3 satellites or air photographs. The SPOT data used at present is derived from the panchromatic (b/w) sensor which has a nominal $10 \mathrm{~m}$ resolution along a $60 \mathrm{~km}$ swath. The data (8-bit unsigned integers) have a range $0-255$ and each scene is 6000 by 6000 pixels.

First both images are displayed and some features identified in each. These are usually road/rail junctions that are evenly distributed across the overlapping parts of each scene. A minimum of 3 such conjugate points are found. The co-ordinates of these points are then found. If suitable (1:10,000 or better) maps are available then these can be used to calculate projected (X, Y, Z in metres) or geographical (i.e. lat. long. ht.) co-ordinates. An alternative is to use the Global Positioning System (GPS) to determine the co-ordinates in the field. These image co-ordinates and their map values are used to constrain the sensor model, a mathematical description of where the satellites were relative to the earth at the time the images were acquired. In addition the sensor model must include information about the relative motion of the satellite over the earth. This is contained in the satellite ephemeris file, automatically read by the program.

The stereo-matcher uses a course to fine resolution pyramid to identify first broad features, and incrementally smaller details common to both images. The user specifies the gridding resolution which is typically 40,20 or $10 \mathrm{~m}$. At $10 \mathrm{~m}$ resolution there can be as many as 36 million conjugate points. This is called a digital disparity model (DDM). The (image) co-ordinates of the matched points are next propagated through the sensor model to produce a random scatter of geographical or projected points that now contain height information. Finally, the matched points are Kriged (Delfiner and Delhomme, 1975) to produce a gridded DEM. The user specifies the number of nearneighbour stations to be used in the Kriging (typically 8 or 16). The more points the smoother the output. Also, the user specifies the search radius so that the interpolation is not performed too far from a matched point.

In some places errors can arise because of mismatches (blunders), leading to elevation errors of omission and commission. These will be flagged by large variances and so they can be filtered out of the interpolated surface. A more serious problem is that of regions for which the matcher could not generate any points in the DDM. This is because no features common to both images could be found. Sometimes this is because the images are genuinely different in these regions, such as snow cover, cloud cover, or change in vegetation or land use. Because of these potential limitations images should be temporally similar (say a few weeks apart) or else should have at least been acquired in the same season. Other regions that may remain unmatched are those that are texturally flat, such as sea areas 
and homogeneous forest or in some cases pasture. However, in most cases about $95 \%$ of possible coverage can be achieved.

\section{Gravimetric Terrain Corrections}

Terrain corrections are really topographic corrections to Bouger corrections. They are always positive and represent the volume integrals of material lying above a station and material in the Bouguer slab below the station that is not present in reality. Terrain corrections can be calculated from elevation data using several methods to varying degrees of accuracy, some of the more advanced methods that have derived and tested are outlined below.

\section{a) Rectangular Prism Method (RPM)}

The gravity effect can be derived analytically for a rectangular prism, such as a cube, which is the basic element of a DEM. The terrain effects of each cube can be summed about a station. The main drawback with this method is that the ground is not terraced in reality, but (except at cliffs) varies smoothly in space. This can lead to errors of as much as 0.2 milligals with slopes of less than $10 \%$, far too great for microgravity surveys, and several milligals in steep mountainous regions.

\section{b) Triangular element method (TEM)}

In reality the gravity effect for any polyhedron can be derived analytically by transforming the volume integral to a surface integral over the bounding facets using Green's theorem. The most basic facet element is a triangular prism. Thus if the surface is divided into triangular regions (by forming diagonals between DEM nodes for example) then we can sum the analytic terrain effects for each element. Although the gravity effect is analytic, usually the integration is performed using Gaussian Quadrature with 3 or 4 quadrature elements per facet. The clear advantage with TEM is that it allows the topography to smoothly vary as a function of height and also it is directly applicable to irregular elevation data via the Delaunay triangulation.

\section{c) Bicubic Rectangular Prism Method (BRPM)}

This describes the basic theory behind the random program T-CORR developed by Rob Davies of TerraDat in May 1997. The drawback that remains with TEM is that in reality not only is terrain a smooth function of location, but so is slope and curvature (1st and 2nd derivatives). Also, where a DEM is available, TEM introduces a doubling in the number of computational elements over RPM. BRPM solves for the bicubic function through each DEM cell such that the function and its first two derivatives are continuous with its neighbours. This means that a smooth terrain is fitted through the data. The problem now is that there is no analytic expression for the terrain effect of a bicubic prism and so a numerical approximation is required. Monte Carlo integration uses pseudo random numbers (PRNs) to effectively generate random co-ordinates corresponding to point masses within each DEM cell. However, this suffers from being slow to converge, and worse, not really representative of masses in the earth. It is for example, entirely possible to generate the same co-ordinate more than once, when in reality, two point masses can never occupy the same space.

The new approach involves progressively filling the cube with points that are evenly spaced, and which never occupy the same space. For each point-mass the question is whether it lies above or below the surface of the cell in question. This is calculated by evaluating the bicubic of the surface at the $x$, and $y$ co-ordinates of the point-mass. This gives the $z$ value of the surface at the $x, y$ coordinates of the mass which is compared with the $z$ co-ordinate of the mass to test whether the mass is above or below the surface. The actual mass of each point is simply the volume of the cell times the density of the Bouguer correction divided by the number of points in each cube. Because of the $r$ squared nature gravity, the number of points-masses should be greatest for cells closest to the gravity 
station, where the topography must be modelled best. This means that the number of points can fall away with the square of the distance and so cells many $\mathrm{km}$ from the station will have only 1 (or zero!) point mass at the centre of the cell. This makes the integration at least as fast as TEM, and much more accurate. Rapid convergence, specifying a maximum of 10,000 points per cell, leads to a terrain correction uncertainty of $<0.0001 \mathrm{mgal}$.

\section{CONCLUSION}

Significant time and cost savings can be achieved through a semi-automated approach to terrain correction calculation. The accuracy of the correction function produced by T-CORR software is greater than that of any field gravimeter used to record the original data. The limiting factors that can affect the accuracy of the final terrain correction have been reduced to the reliability of assumed densities and the spatial resolution of elevation data. The use of the advanced stereo-matcher algorithm enables creation of a complete coverage regional DEM model at higher spatial resolution than could be achieved through conventional $1: 10,000$ map digitising work.

In areas of irregular topography the use of the described methodology for calculating terrain corrections can make the difference between successful identification of target features and failure. In addition, calculation of a terrain correction map for a planned study region can enable optimal positioning of the gravity stations to reduce the effects of any potential error in elevation data and density assumptions to a minimum.

Delfiner P, and J.P. Delhomme, 1975. Optimum interpolation by Kriging, in J.C. Davis and M.J. McCullagh, Eds, Display and Analysis of Spatial Data, pp 96-114, John Wiley and Sons.

Hammer, S., 1982. Critique of terrain corrections for gravity stations. Geophysics 47, 839-40.

O'Neill M.A. and Denos M.I., 1996. Automated system for coarse-to-fine pyramidal area correlation stereo matching, Image and Vis. Comp. 14(3): 225-236. 